\title{
Comparison of Predictors of COVID-19 Vaccination Intention Between Healthcare Workers and Non-Healthcare Workers in China
}

\author{
Lisha Chi ${ }^{1, *}$ \\ Guojing Zhao $\mathbb{D}^{2, *}$ \\ Naiche Chen ${ }^{3, *}$ \\ Guanghui Shen ${ }^{4}$ \\ Kai Huang ${ }^{4}$ \\ Xiaoyu Xia' \\ Yijing Chen' \\ Jian Liu' \\ Ran $X u^{5}$ \\ Yanhan Chen ${ }^{6}$ \\ Weijie Dong $^{7}$ \\ Jiexia Zheng ${ }^{8}$
}

'School of Pharmaceutical Science, Wenzhou Medical University, Wenzhou, People's Republic of China; ${ }^{2}$ Institute of China Innovation and Entrepreneurship Education, Wenzhou Medical University, Wenzhou, People's Republic of China; ${ }^{3}$ Renji College, Wenzhou Medical University, Wenzhou, People's Republic of China; ${ }^{4}$ School of Mental Health, Wenzhou Medical University, Wenzhou, People's Republic of China; ${ }^{5}$ Department of Natural Science, Affiliated School of Wenzhou University Town, Wenzhou, People's Republic of China; 'School of Stomatology, Wenzhou Medical University, Wenzhou, People's Republic of China; ${ }^{7}$ The I th School of Medicine, Wenzhou Medical University, Wenzhou, People's Republic of China; ${ }^{8}$ Department of Student Affairs, Wenzhou Medical University, Wenzhou, People's Republic of China

*These authors contributed equally to this work

Correspondence: Jiexia Zheng Department of Student Affairs, Wenzhou Medical University, Wenzhou, Zhejiang Province, People's Republic of China Email zhengjiexia202I@।63.com
Background: Vaccination is an effective strategy to mitigate the spread of COVID-19. This study aimed to compare predictors of vaccination intention between healthcare workers (HCWs) and non-healthcare workers (non-HCWs) in China.

Methods: A web-based cross-sectional survey was conducted among HCWs and non-HCWs. Several bivariate analysis techniques, eg, crosstab with Chi-square, independent $t$-test and single factor ANOVA, were performed to analyze the correlation. After that, a series of multivariate binary regressions were employed to determine predictors of vaccination intention.

Results: Intention was closely and significantly related with gender, perceived vaccination knowledge, perceived importance and effectiveness of vaccine to prevent COVID-19. HCWs and non-HCWs were heterogeneous, since vaccination intention, perceived knowledge, and attitudes (eg, importance, severity, risk) toward COVID-19 or vaccine had statistically significant difference between the two groups. With comparison of predictors of vaccination intention, for HCWs, demographic factors were the major predictors of COVID-19 vaccination intention. Female HCWs and HCWs with a Master's or higher degree were more hesitant about vaccination ( $\mathrm{P}=0.01$ and $\mathrm{P}<0.001$, respectively), while HCWs had greater vaccination intention as their age increased $(\mathrm{P}=0.02)$. For non-HCWs, perceived vaccination knowledge was the major predictor of COVID-19 vaccination intention $(\mathrm{P}<0.001)$. Additionally, perceived importance and effectiveness of vaccine were predictors for both HCWs and non-HCWs.

Conclusion: Vaccination intention of HCWs was greater than that of non-HCWs in China. Measures should be taken to improve the vaccination rate based on the predictors of vaccination intention identified in this study. For HCWs, especially those with a high level of education or who were females, the safety and effectiveness of vaccines in use may reinforce their vaccination intention. For non-HCWs, popularization of general medical knowledge, including of vaccinepreventable diseases, may increase their vaccination intention.

Keywords: COVID-19 vaccine, intention, predictor, HCWs, non-HCWs

\section{Introduction}

COVID-19 (also known as novel coronavirus pneumonia) was first reported more than a year ago and now affects nearly every country in the world. The cumulative number of COVID-19 cases globally surpassed 200 million on August 5, 2021, and reached 100 million in just 6 months. ${ }^{1}$

Vaccination remains one of the most effective strategies to control epidemics. However, promoting vaccination can be a daunting challenge for local governments. ${ }^{2}$ Following the launch of a COVID-19 vaccine in December 2020, studies evaluated the 
intention of the public to receive COVID-19 vaccines in various countries. In total, $69 \%$ and $55.8 \%$ of participants were willing to be vaccinated in the United States and England, respectively. ${ }^{3,4}$ A nationwide online survey reported that $28.7 \%, 54.6 \%$, and $16.7 \%$ of respondents definitely intended, probably intended, and did not intend to be vaccinated against COVID-19 in China, respectively. ${ }^{5}$ Vaccination intention varied by time, region and public perception. ${ }^{6}$ Better understanding of the intention of the public to receive a COVID-19 vaccine and key predictors of this intention may help policymakers adopt measures to improve vaccination coverage.

When a new vaccine was launched, there were several predictors of vaccination, including perceptions of disease risk, vaccine safety and efficacy, and vaccination knowledge. ${ }^{7-11}$ An Irish study reported that medical workers were reluctant to receive a seasonal flu vaccine because of doubts about its effectiveness and a lack of trust. ${ }^{11}$ Many Beijing residents were skeptical about the effectiveness of the H7N9 vaccine and did not worry about the risk of getting ill, and these were predictors of their intention to receive this vaccine. ${ }^{12}$ A survey in the United Arab Emirates showed that vaccine safety, side effects, and receiving too many injections were key predictors of childhood vaccine hesitancy. ${ }^{13}$ A study in the United States indicated that the strongest predictors of whether people received a COVID-19 vaccine were trust in the system evaluating vaccines and perception of local COVID-19 vaccination norms. ${ }^{14}$ Assessment of these factors may help develop intervention methods and policies to improve vaccination coverage. The key predictors of the intention of the public to receive a COVID-19 vaccine must be analyzed. Such information may help policymakers take steps to improve vaccination coverage.

A study in China reported that COVID-19 vaccination intention was greater among people engaged in medicalrelated industries than among those who were not. ${ }^{15} \mathrm{HCWs}$ were the most reliable source of information and had the greatest positive impact on public vaccination. ${ }^{16-18} \mathrm{HCWs}$ were the leading advocate of immunization, and therefore their intention to receive a vaccine can improve public acceptance of vaccination, reduce reluctance about vaccination, and guide informed decisions about vaccination. ${ }^{19,20}$ For example, the public in the United States were more willing to be vaccinated if they thought their healthcare providers would be vaccinated. ${ }^{21}$ However, little was known about the differences or similarities of predictors of the intention of HCWs and non-HCWs to receive a COVID-19 vaccine.
Therefore, this study compared predictors of COVID19 vaccination intention between HCWs and non-HCWs in terms of demographics, knowledge, and attitudes. The findings of this study will help policymakers develop strategies targeted to different populations according to predictors of vaccination intention in order to improve COVID-19 vaccination coverage.

\section{Methods}

\section{Study Design and Setting}

This survey was conducted in December 2020, when a COVID-19 vaccine first became available in China. A cross-sectional survey was designed using a survey program based on a social media platform (WeChat) called "Questionnaire Star", which provides functions equivalent to Amazon Mechanical Turk, and used a snowball sampling strategy. Respondents were divided into two groups: HCWs and non-HCWs. According to occupation category, HCWs consisted of clinicians, nurses and laboratorians, and non-HCWs consisted of administrative personnel, teaching staffs and business and service workers, etc. The investigator sent the survey link to the main contact (aged 18 years and older) via social media (Questionnaire Star app) and asked them to conduct further investigations. After clicking the link, respondents were directed to the informed consent page and then completed the questionnaire.

\section{Study Sample}

The sample size was calculated using the following formula: $\mathrm{N}=\frac{\mu_{\alpha}^{2}}{\delta^{2}} P(1-P)$, where $\alpha=0.01$ and $\mu_{\alpha}=2.576$. The estimated acceptable margin of error for proportion $\mathrm{d}$ was 0.05 . According to previous studies, the sample rate $\mathrm{P}=0.15$. The formula estimated the sample size to be 338 . For subgroup analyses, the final sample size was set to 820 , including $410 \mathrm{HCWs}$ and 410 non-HCW. After excluding invalid responses of $23 \mathrm{HCWs}$ and 4 nonHCWs, 387 HCWs and 406 non-HCWs were included in the study.

\section{Questionnaire Development}

We determined the focus of this survey by consulting the literature and designed a draft questionnaire. The questions related to (1) demographics, including personal details such as gender, age, education level, and occurrence of cardiovascular or chronic diseases; (2) knowledge of COVID-19 vaccination including perceived 
knowledge of COVID-19 vaccine-preventable diseases; (3) attitudes toward COVID-19 vaccination including perceptions about the importance of COVID-19 vaccination to prevent disease, effectiveness of the COVID-19 vaccine, severity of COVID-19, and risk of COVID-19; and (4) intention to receive the COVID-19 vaccine. The latter was assessed by asking respondents whether they would like to get the COVID-19 vaccine as soon as possible after it became available. The response options were "Accept", "Hesitance", and "Reject". The reliability of the questionnaire was confirmed according to the internal consistency (Cronbach's alpha coefficient $>0.70$ ). The options for the questionnaire items were based on a 4-point scale $^{22}$ [4 = "Strongly Agree", 3 = "Agree", 2 = "Disagree", and 1 = "Strongly Disagree"]. We tried to keep the questionnaire short so that it could be completed quickly and was easy to understand.

\section{Data Analysis}

Basic descriptive statistics were used to describe all variables. As designed, Chi-square test was employed to compare demographics among different COVID-19 vaccination intentions and to compare demographics and COVID-19 vaccination intention between HCWs and nonHCWs. The single-factor ANOVA was performed to compare the mean scores of knowledge and attitude variables among different COVID-19 vaccination intentions. The independent $t$-test was used to compare the mean scores of knowledge and attitude variables between HCWs and non-HCWs. Then, a series of multivariate binary logistic regressions were performed to determine the effect of independent variables on our outcome variables as a dummy measure of intention to receive a COVID-19 vaccine, ie, hesitance vs accept and reject vs accept in HCWs and non-HCWs groups separately. Odds ratios (OR) and their 95\% confidence intervals (95\% CI) were reported and used to analyze the effect of independent variables on our outcome variables. All analyses were performed using SPSS 20.0 (IBM Corp, Armonk, NY, USA). A p-value $<0.05$ was considered statistically significant.

\section{Results}

Demographic information was provided in Tables 1 and 2 where the former one added the comparison of demographics among different COVID-19 vaccination intentions and the latter one did the comparison of demographics and COVID-19 vaccination intention between HCWs and non-HCWs. A total of 793 valid responses were received with $387 \mathrm{HCWs}$ and 406 nonHCWs separately. The intentions to receive a COVID-19 vaccine were separated into three groups among which 450 respondents were willing to accept the vaccine, 110 to reject, and the rest 233 still hesitant.

\section{Intention to Receive a COVID-19 Vaccine}

As Table 1 shows, for all the respondents, the proportions of males and females were $43.3 \%$ and $56.7 \%$ among accept group respectively, $33.3 \%$ and $66.4 \%$ among reject group respectively, and $29.6 \%$ and $70.4 \%$ among hesitance group, respectively. Additionally, the Chi-square indicated that there was a statistically significant correlation between gender distribution and the distribution of intentions to receive a COVID-19 vaccine. We grouped our respondents into four groups based on their age, ie, 18 25 group, 26 35 group, 36 45 group, and above 45 group, and there was not statistically significant correlation between age distribution and the distribution of vaccination intentions. We could also tell the distribution of education status and the occurrence of cardiovascular or chronic diseases were not statistically significant correlation with vaccination intention. As the knowledge of and attitudes toward COVID-19 vaccination, an F-value of 41.99 with $\mathrm{P}<0.001$ indicating perceived knowledge of COVID-19 vaccine-preventable diseases was statistically significant correlated with intention to receive a COVID-19 vaccine. For the rest two, we had an F-value of 76.21 with $\mathrm{P}<0.001$ for perceptions about the importance of COVID-19 vaccination to prevent disease and an F-value of 61.19 with $\mathrm{P}<0.001$ for the perception of effectiveness of COVID-19 vaccination. However, perception concerning severity and risk of COVID-19 were not statistically significant correlated with intention to receive a COVID-19 vaccine.

As our topic is more on a detailed image of the differences of HCWs and non-HCWs than the overall image. We also compared the demographics and COVID-19 vaccination intention between HCWs and non-HCWs. As shown in Table 2, the proportions of respondents who wanted to receive a COVID-19 vaccine as soon as possible, did not want to receive a COVID-19 vaccine, and who were hesitant about receiving a COVID-19 vaccine were $64.6 \%, 14.2 \%$, and $21.2 \%$ for $\mathrm{HCWs}$, respectively, and $49.3 \%, 13.5 \%$, and $37.2 \%$ for non-HCWs, respectively. A higher proportion of HCWs than non-HCWs intended to receive a COVID-19 vaccine $(\mathrm{P}<0.001)$. For the differences in the perceived knowledge and attitudes of 
Table I Comparison of Demographics, Knowledge, and Attitudes Toward COVID-19 Vaccination Among Three Groups of Intent to COVID-I 9 Vaccination

\begin{tabular}{|c|c|c|c|c|c|c|}
\hline & $\begin{array}{c}\text { Total } \\
\text { n (\%)/M } \mathbf{M S D}\end{array}$ & $\begin{array}{c}\text { Accept } \\
\text { n (\%)/M } \pm \text { SD }\end{array}$ & $\begin{array}{c}\text { Reject } \\
\text { n (\%)/M } \pm \text { SD }\end{array}$ & $\begin{array}{c}\text { Hesitance } \\
\text { n (\%)/M } \pm \text { SD }\end{array}$ & $\chi 2 / F$ & $\mathbf{P}$ \\
\hline \multicolumn{7}{|l|}{ I. Demographic characteristics } \\
\hline \multicolumn{7}{|l|}{ Gender } \\
\hline Male & 301 (38.0\%) & $195(43.3 \%)$ & 37 (33.6\%) & 69 (29.6\%) & 13.28 & 0.013 \\
\hline Female & $492(62.0 \%)$ & $255(56.7 \%)$ & $73(66.4 \%)$ & $164(70.4 \%)$ & & \\
\hline \multicolumn{7}{|l|}{ Age (years) } \\
\hline $18-25$ & 209 (26.4\%) & $109(24.2 \%)$ & $30(27.3 \%)$ & $70(30 \%)$ & 8.74 & 0.189 \\
\hline $26-35$ & $228(28.8 \%)$ & $122(27.1 \%)$ & $33(30 \%)$ & $73(31.3 \%)$ & & \\
\hline $36-45$ & $268(33.8 \%)$ & $159(35.3 \%)$ & 37 (33.6\%) & $72(30.9 \%)$ & & \\
\hline Above 45 & $88(11.1 \%)$ & $60(13.3 \%)$ & $10(9.1 \%)$ & 18 (7.7\%) & & \\
\hline \multicolumn{7}{|l|}{ Education status } \\
\hline College degree and lower & $126(15.9 \%)$ & $81(18 \%)$ & $13(11.8 \%)$ & $32(13.7 \%)$ & 5.74 & 0.219 \\
\hline Undergraduate degree & $483(60.9 \%)$ & $273(60.7 \%)$ & 72 (65.5\%) & 138 (59.2\%) & & \\
\hline Master's degree and higher & $184(23.2 \%)$ & $96(21.3 \%)$ & $25(22.7 \%)$ & $63(27 \%)$ & & \\
\hline \multicolumn{7}{|l|}{ Have cardiovascular diseases or chronic diseases } \\
\hline Yes & $58(7.3 \%)$ & $38(8.4 \%)$ & $10(9.1 \%)$ & $10(4.3 \%)$ & 4.50 & 0.105 \\
\hline No & $735(92.7 \%)$ & $412(9 \mid .6 \%)$ & $100(90.9 \%)$ & $223(95.7 \%)$ & & \\
\hline \multicolumn{7}{|l|}{ 2. Knowledge of COVID-I9 vaccination } \\
\hline $\begin{array}{l}\text { Perceived knowledge of COVID-19 vaccine- } \\
\text { preventable diseases }\end{array}$ & - & $2.85 \pm 0.71$ & $2.59 \pm 0.68$ & $2.36 \pm 0.59$ & 41.99 & $<0.001$ \\
\hline \multicolumn{7}{|l|}{ 3. Attitudes toward COVID-19 vaccination } \\
\hline $\begin{array}{l}\text { Perception that COVID-19 vaccination is an } \\
\text { important means to prevent disease }\end{array}$ & - & $3.47 \pm 0.53$ & $2.85 \pm 0.74$ & $3.06 \pm 0.53$ & 76.21 & $<0.001$ \\
\hline Perception that the COVID-19 vaccine is effective & - & $3.32 \pm 0.54$ & $2.77 \pm 0.69$ & $2.96 \pm 0.51$ & 61.19 & $<0.001$ \\
\hline Perception that COVID-19 is a severe illness & - & $3.44 \pm 0.61$ & $3.38 \pm 0.62$ & $3.42 \pm 0.58$ & 0.41 & 0.661 \\
\hline Perception that COVID-19 is a risk & - & $2.47 \pm 0.70$ & $2.35 \pm 0.74$ & $2.42 \pm 0.64$ & 1.70 & 0.184 \\
\hline
\end{tabular}

respondents toward COVID-19 vaccination, perceived knowledge of COVID-19 vaccine-preventable diseases was higher among HCWs than among non-HCWs $(\mathrm{P}<$ 0.001). HCWs had more positive attitudes toward COVID19 vaccination than non-HCWs, including the perceptions about the importance of COVID-19 vaccination to prevent disease ( $P=0.01)$, severity of COVID-19 $(P=0.004)$ and risk of COVID-19 $(\mathrm{P}<0.001)$ than non-HCWs. Perceived effectiveness of the COVID-19 vaccine did not significantly differ between HCWs and non-HCWs $(\mathrm{P}=0.22)$.
Taking the two comparisons concerning the severity and risk of COVID-19, first among various intentions to receive a COVID-19 vaccine and second between HCWs and nonHCWs into consideration, it might be possible that HCWs and non-HCWs were heterogeneous. Hence, it would be suitable to test the effect of independent variables on the dependent variables in HCWs and non-HCWs separately.

Additionally, there were also significant differences in the proportions of demographics. The proportions of males and females were $31.8 \%$ and $68.2 \%$ among HCWs, 
Table 2 Comparison of Demographics, Knowledge of and Attitudes Toward COVID-19 Vaccination, and Vaccination Intention Between HCWs and Non-HCWs

\begin{tabular}{|c|c|c|c|c|c|}
\hline & $\begin{array}{c}\text { Total n (\%)/ } \\
\text { M } \pm \text { SD }\end{array}$ & $\begin{array}{l}\text { HCWs n (\%)l } \\
\text { M } \pm \text { SD }\end{array}$ & $\begin{array}{l}\text { Non-HCWs } \\
\text { n (\%)/M } \pm S D\end{array}$ & $\chi^{2 / t}$ & $\mathbf{P}$ \\
\hline \multicolumn{6}{|l|}{ I. Demographic characteristics } \\
\hline \multicolumn{6}{|l|}{ Gender } \\
\hline Male & 301 (38.0\%) & $123(31.8 \%)$ & $178(43.8 \%)$ & 12.24 & $<0.001$ \\
\hline Female & $492(62.0 \%)$ & $264(68.2 \%)$ & $228(56.2 \%)$ & & \\
\hline \multicolumn{6}{|l|}{ Age (years) } \\
\hline $18-25$ & $209(26.4 \%)$ & 118 (30.5\%) & 91 (22.4\%) & 10.59 & 0.014 \\
\hline $26-35$ & $228(28.8 \%)$ & $108(27.9 \%)$ & $120(29.6 \%)$ & & \\
\hline $36-45$ & $268(33.8 \%)$ & $129(33.3 \%)$ & $139(34.2 \%)$ & & \\
\hline Above 45 & $88(11.1 \%)$ & $32(8.3 \%)$ & $56(13.8 \%)$ & & \\
\hline \multicolumn{6}{|l|}{ Education status } \\
\hline College degree and lower & $126(15.9 \%)$ & $40(10.3 \%)$ & $86(21.2 \%)$ & 23.10 & 0.001 \\
\hline Undergraduate degree & $483(60.9 \%)$ & $265(68.5 \%)$ & $218(53.7 \%)$ & & \\
\hline Master's degree and higher & I84 (23.2\%) & $82(21.2 \%)$ & $102(25.1 \%)$ & & \\
\hline \multicolumn{6}{|l|}{ Have cardiovascular diseases or chronic diseases } \\
\hline Yes & $58(7.3 \%)$ & $26(6.7 \%)$ & $32(7.9 \%)$ & 0.40 & 0.31 \\
\hline No & 735 (92.7\%) & $361(93.3 \%)$ & $374(92.1 \%)$ & & \\
\hline \multicolumn{6}{|l|}{ 2. Knowledge of COVID-19 vaccination } \\
\hline $\begin{array}{l}\text { Perceived knowledge of COVID-19 vaccine-preventable } \\
\text { diseases }\end{array}$ & - & $2.86 \pm 0.66$ & $2.49 \pm 0.71$ & 7.70 & $<0.001$ \\
\hline \multicolumn{6}{|l|}{ 3. Attitudes toward COVID-19 vaccination } \\
\hline $\begin{array}{l}\text { Perception that COVID-19 vaccination is an important } \\
\text { means to prevent disease }\end{array}$ & - & $3.32 \pm 0.62$ & $3.21 \pm 0.61$ & 2.50 & 0.01 \\
\hline Perception that the COVID-19 vaccine is effective & - & $3.17 \pm 0.61$ & $3.12 \pm 0.58$ & 1.24 & 0.22 \\
\hline Perception that COVID-19 is a severe illness & - & $3.49 \pm 0.61$ & $3.36 \pm 0.59$ & -2.89 & 0.004 \\
\hline Perception that COVID-19 is a risk & - & $2.58 \pm 0.66$ & $2.30 \pm 0.69$ & 5.90 & $<0.001$ \\
\hline \multicolumn{6}{|l|}{ 4. Intention to receive a COVID-19 vaccine } \\
\hline Accept & $450(56.7 \%)$ & $250(64.6 \%)$ & 200 (49.3\%) & 25.55 & $<0.001$ \\
\hline Reject & 110 (13.9\%) & 55 (I4.2\%) & 55 (13.5\%) & & \\
\hline Hesitance & 233 (29.4\%) & $82(21.2 \%)$ & 151 (37.2\%) & & \\
\hline
\end{tabular}

respectively, and $43.8 \%$ and $56.2 \%$ among non-HCWs, for $68.5 \%$ of $\mathrm{HCWs}$ and $53.7 \%$ of non-HCWs and respectively. Age ranged from 18 years to older than 45 a Master's or higher degree for more than $20 \%$ of both years. The education level was an undergraduate degree HCWs and non-HCWs. The occurrence of cardiovascular 
or chronic diseases did not significantly differ between HCWs and non-HCWs.

\section{Comparison of the Predictors of COVID-19 Vaccination Intention}

A series of multivariate binary logistic regressions were used to evaluate predictors of vaccination intention (Table 3). (1) The different predictors: For HCWs, demographic factors (gender, education level and age) were major predictors of COVID-19 vaccination intention. Female HCWs were more hesitant about receiving a COVID-19 vaccine than male HCWs (Hesitance vs Accept: OR $=0.28,95 \% \mathrm{CI}=0.12$ $0.68, \mathrm{P}=0.01)$. HCWs with a Master's or higher degree were more hesitant about receiving a COVID-19 vaccine (Hesitance vs Accept: $\mathrm{OR}=0.08,95 \% \mathrm{CI}=0.02-0.38, \mathrm{P}<$ $0.001)$. HCWs had greater vaccination intention as their age increased (Hesitance vs Accept: $\mathrm{OR}=10.65,95 \% \mathrm{CI}=1.49$ $76.18, \mathrm{P}=0.02$ ). For non-HCWs, perceived knowledge of COVID-19 vaccination was the major predictor of COVID19 vaccination intention. Non-HCWs with good perceived knowledge of COVID-19 vaccine-preventable diseases were 2.35 times more likely to intend to be vaccinated than their counterparts (Hesitance vs Accept: $\mathrm{OR}=2.35,95 \% \mathrm{CI}=$ 1.60-3.45, P <0.001). (2) The similar predictors: Attitudes toward COVID-19 vaccination were a predictor of COVID19 vaccination intention for both HCWs and non-HCWs. Perceptions about the importance of COVID-19 vaccination to prevent disease (Hesitance vs Accept: $\mathrm{OR}=6.24,95 \% \mathrm{CI}$ $=2.49-15.60, \mathrm{P}<0.001$; Reject vs Accept: $\mathrm{OR}=2.39,95 \%$ $\mathrm{CI}=1.20-4.74, \mathrm{P}=0.01)$ and effectiveness of the COVID-19 vaccine (Hesitance vs Accept: $\mathrm{OR}=3.37,95 \% \mathrm{CI}=1.32$ 8.57, $\mathrm{P}=0.01$; Reject vs Accept: $\mathrm{OR}=2.48,95 \% \mathrm{CI}=1.19$ $5.16, \mathrm{P}=0.02$ ) were predictors of positive vaccination intention for HCWs. Similarly, perceptions about the importance of COVID-19 vaccination to prevent disease (Hesitance vs Accept: $\mathrm{OR}=2.11,95 \% \mathrm{CI}=1.22-3.64, \mathrm{P}=0.01$; Reject vs Accept: $\mathrm{OR}=5.83,95 \% \mathrm{CI}=2.33-14.58, \mathrm{P}<0.001)$ and effectiveness of the COVID-19 vaccine (Reject vs Accept: $\mathrm{OR}=2.29,95 \% \mathrm{CI}=0.96-5.47, \mathrm{P}=0.03$ ) were predictors of positive vaccination intention for non-HCWs. Meanwhile, perceived severity of COVID-19 and risk of COVID-19 did not predict the intention of $\mathrm{HCWs}$ or non-HCWs to receive a COVID-19 vaccine.

\section{Discussion}

Following the successful development of COVID-19 vaccines, studies had investigated acceptance of these vaccines. ${ }^{3,5,23,24}$ In this study, we compared predictors of COVID-19 vaccination intention between HCWs and nonHCWs in terms of demographics, knowledge, and attitudes. In total, $387 \mathrm{HCWs}$ and 406 non-HCWs were included in our study. A higher proportion of HCWs than non-HCWs intended to receive a COVID-19 vaccine ( $\mathrm{P}<$ 0.001). Perceived knowledge of HCWs, including ancillary HCWs (such as nurses and laboratory technicians), about COVID-19 vaccine-preventable diseases was significantly better than that of non-HCWs $(\mathrm{P}<0.001)$. A study from the United States found that knowledge of COVID19 vaccination differed between clinical decision-makers (such as doctors) and the general public. ${ }^{25}$ Interestingly, ancillary HCWs in China appeared to have better perception of knowledge of COVID-19 vaccination relative to the public than their American peers. In addition, the mean scores $( \pm \mathrm{SD})$ of positive attitudes were higher among HCWs than among non-HCWs, including perceptions about the importance of COVID-19 vaccination to prevent disease $(P=0.01)$, severity of COVID-19 $(P=0.004)$ and risk of COVID-19 ( $\mathrm{P}<0.001)$, except for perception of vaccine effectiveness $(\mathrm{P}=0.22)$. This was consistent with a previous study about the knowledge of HCWs and their attitudes toward the influenza vaccine in Jordan. ${ }^{26}$

We compared predictors of intention to receive a COVID-19 vaccine between HCWs and non-HCWs. Female HCWs tended to be more hesitant about receiving a COVID-19 vaccine than male HCWs $(\mathrm{P}=0.01)$. A similar study of HCWs in Saudi Arabia showed that the intention of men to be vaccinated is nearly 1.5 times higher than that of women. ${ }^{27}$ This may be because females were more concerned about vaccines and diseases than males and thus were more concerned about the safety and effectiveness of vaccines. ${ }^{28} \mathrm{HCWs}$ were more willing to receive a COVID-19 vaccine as their age increased. Similarly, a study in France reported that older age correlated with COVID-19 vaccination intention. ${ }^{29}$

Surprisingly, perceived knowledge of COVID-19 vaccination was a predictor of vaccination intention for nonHCWs ( $<<0.001)$, but not for HCWs. The more perceived knowledge of COVID-19 vaccine-preventable diseases that non-HCWs had, the more likely they were to intend to be vaccinated. Consistently, a previous study reported that lack of knowledge about vaccine-preventable diseases was a key factor responsible for the decline of vaccine coverage among the general public. ${ }^{30}$ Thus, increasing medical general knowledge (such as of vaccine-preventable diseases) was 
Table 3 Comparison of Predictors of COVID-19 Vaccination Intention Between HCWs and Non-HCWs

\begin{tabular}{|c|c|c|c|c|c|c|c|c|}
\hline & \multicolumn{4}{|c|}{ Healthcare Workers (HCWs) } & \multicolumn{4}{|c|}{ Non-Healthcare Workers (Non-HCWs) } \\
\hline & \multicolumn{2}{|c|}{$\begin{array}{l}\text { Intent to Be Vaccinated: } \\
\text { Hesitance vs Accept }\end{array}$} & \multicolumn{2}{|c|}{$\begin{array}{l}\text { Intent to Be Vaccinated: } \\
\text { Reject vs Accept }\end{array}$} & \multicolumn{2}{|c|}{$\begin{array}{l}\text { Intent to Be Vaccinated: } \\
\text { Hesitance vs Accept }\end{array}$} & \multicolumn{2}{|c|}{$\begin{array}{c}\text { Intent to Be Vaccinated: Reject } \\
\text { vs Accept }\end{array}$} \\
\hline & OR $(95 \% \mathrm{Cl})$ & $\mathbf{P}$ & OR $(95 \% \mathrm{Cl})$ & $\mathbf{P}$ & OR $(95 \% \mathrm{Cl})$ & $\mathbf{P}$ & OR (95\% Cl) & $\mathbf{P}$ \\
\hline \multicolumn{9}{|l|}{ I. Demographic characteristic } \\
\hline \multicolumn{9}{|l|}{ Gender } \\
\hline Male & I (ref) & - & I (ref) & - & I (ref) & - & I (ref) & - \\
\hline Female & $0.28(0.12,0.68)$ & 0.01 & $0.55(0.25,1.23)$ & 0.15 & $0.85(0.52,1.39)$ & 0.52 & $1.01(0.48,2.12)$ & 0.97 \\
\hline \multicolumn{9}{|l|}{ Age (years) } \\
\hline $18-25$ & I (ref) & - & I (ref) & - & I (ref) & - & I (ref) & - \\
\hline $26-35$ & $1.16(0.5 \mathrm{I}, 2.67)$ & 0.72 & $0.54(0.23,1.28)$ & 0.16 & $1.06(0.55,2.04)$ & 0.87 & $1.75(0.59,5.21)$ & 0.31 \\
\hline $36-45$ & $2.37(0.93,6.03)$ & 0.07 & $1.36(0.50,3.75)$ & 0.55 & $0.74(0.39,1.39)$ & 0.35 & $1.20(0.48,3.03)$ & 0.69 \\
\hline Above 45 & $10.65(1.49,76.18)$ & 0.02 & $1.13(0.27,4.63)$ & 0.87 & $1.60(0.66,3.85)$ & 0.30 & $2.45(0.57,10.47)$ & 0.23 \\
\hline \multicolumn{9}{|l|}{ Education status } \\
\hline College degree and lower' & I (ref) & - & I (ref) & - & I (ref) & - & I (ref) & - \\
\hline Undergraduate degree & $0.28(0.07,1.12)$ & 0.07 & $0.60(0.20,1.78)$ & 0.36 & $0.87(0.44,1.71)$ & 0.68 & $0.51(0.16,1.57)$ & 0.24 \\
\hline Master's degree and higher & $0.08(0.02,0.38)$ & $<0.001$ & $0.43(0.12,1.56)$ & 0.20 & $0.52(0.23,1.16)$ & 0.11 & $0.41(0.10,1.64)$ & 0.21 \\
\hline \multicolumn{9}{|l|}{ Have cardiovascular diseases or chronic diseases } \\
\hline Yes & I (ref) & - & I (ref) & - & I (ref) & - & I (ref) & - \\
\hline No & $0.87(0.09,9.68)$ & 0.91 & $1.98(0.50,7.90)$ & 0.36 & $0.61(0.25,1.5 \mathrm{I})$ & 0.29 & $1.19(0.34,4.15)$ & 0.79 \\
\hline \multicolumn{9}{|l|}{ 2. Knowledge of COVID-19 vaccination } \\
\hline Perceived knowledge of COVID-19 vaccine-preventable diseases & $1.79(0.98,3.30)$ & 0.06 & I.44 $(0.77,2.68)$ & 0.26 & $2.35(1.60,3.45)$ & $<0.001$ & $0.93(0.53,1.61)$ & 0.79 \\
\hline \multicolumn{9}{|l|}{ 3. Attitudes toward COVID-19 vaccination } \\
\hline Perception that COVID-19 vaccination is an important means to prevent disease & $6.24(2.49,15.60)$ & $<0.001$ & $2.39(1.20,4.74)$ & 0.01 & $2.11(1.22,3.64)$ & 0.01 & $5.83(2.33,14.58)$ & $<0.001$ \\
\hline Perception that the COVID-19 vaccine is effective & $3.37(1.32,8.57)$ & 0.01 & $2.48(1.19,5.16)$ & 0.02 & $1.19(0.69,2.05)$ & 0.53 & $2.29(0.96,5.47)$ & 0.03 \\
\hline Perception that COVID-19 is a severe illness & $1.08(0.61,1.90)$ & 0.80 & $1.21(0.68,2.17)$ & 0.52 & $0.88(0.59,1.33)$ & 0.55 & $0.59(0.32,1.07)$ & 0.08 \\
\hline Perception that COVID-19 is a risk & $0.74(0.43,1.25)$ & 0.25 & $0.96(0.57,1.63)$ & 0.89 & $1.05(0.73,1.52)$ & 0.78 & $1.62(0.96,2.75)$ & 0.07 \\
\hline
\end{tabular}


an effective measure to increase COVID-19 vaccine uptake among non-HCWs.

However, perceived knowledge of COVID-19 vaccinepreventable diseases did not predict the vaccination intention of HCWs. HCWs who were more highly educated (especially those with a Master's or higher degree) were more hesitant about receiving a COVID-19 vaccine $(\mathrm{P}<0.001)$. Highly educated HCWs with more professional medical knowledge were more concerned about the vaccine development process. ${ }^{31,32}$ They were aware that the development of human vaccines can take years or even decades and involves animal testing, multi-phase clinical trials, new drug applications, and real-world studies. By contrast, COVID-19 vaccines have been developed and mass vaccination campaigns have been rolled out at incredible speed. This accelerated research process raises doubts in HCWs about the safety and efficacy of COVID-19 vaccines. ${ }^{33}$ A study by Barry et al reported that $\mathrm{HCWs}$ were $60 \%$ less willing to accept COVID-19 vaccines if they were hastily administered without evidence-based testing. ${ }^{27}$ A recent study from the United States provided a detailed explanation of COVID-19 vaccine development, which drew attention from the medical community and may reduce doubts about COVID-19 vaccines among HCWs. ${ }^{34}$ As CBS News reported

We have a lot more work to do to get HCWs to take the vaccine. Simply making it available is not enough. We have to take a more precise, targeted approach to reach different segments of population to overcome hesitancy. ${ }^{35}$

As early as October, 2020, Sarah Kreps et al used the hypothetical vaccine method to survey vaccine preference in the United States and showed that vaccine efficacy was the most important factor associated with intention to receive COVID-19 vaccines. ${ }^{36}$ Our survey confirmed that perceptions about the importance of COVID-19 vaccination to prevent disease $(\mathrm{P}<0.001)$ and effectiveness of the COVID-19 vaccine $(\mathrm{P}<0.05)$ were predictors of COVID19 vaccine intention among HCWs and non-HCWs. Meanwhile, perceived severity of COVID-19 and risk of COVID-19 did not predict the intention of HCWs or nonHCWs to receive a COVID-19 vaccine. This was consistent with Faasse et al findings in an Australian sample that perceived risk and severity of infection were only marginally associated with intentions to get a vaccine. ${ }^{37}$ Predictors of intention to receive a COVID-19 vaccine were complex, and cultural, social, and political differences between countries should be considered during the vaccination decisionmaking process. ${ }^{6}$ For example, a study from the United
States showed that the strongest predictors of vaccination intention were trust in the system evaluating vaccines and local COVID-19 vaccination norms. ${ }^{14}$ This was different from the findings of the current study, which was conducted in China, where the public had a high degree of trust in the government. ${ }^{38}$

This study had some limitations. Firstly, the data were collected through an electronic, rather than face-to-face questionnaire, and the questionnaire was completed on a voluntary basis. This may have resulted in sampling bias and uncontrollable situations during completion of the questionnaire. Due to the relatively small sample size, the results should be considered preliminary. Secondly, the survey was performed at a specific point in time during the pandemic, and the results may change as vaccines control the epidemic.

\section{Conclusion}

In China, intention to receive a COVID-19 vaccine was higher among HCWs than among non-HCWs. In addition, HCWs had better perceived knowledge of COVID-19 vaccination and more positive attitudes toward COVID-19 vaccination, including perceptions about the importance of COVID-19 vaccination to prevent disease, severity and risk of COVID-19 than non-HCWs. Measures should be taken to improve the vaccination rate based on the predictors of vaccination intention identified in this study. For HCWs, the hesitancy of highly educated or female HCWs about vaccination must be addressed. They may be concerned about the safety and effectiveness of rapidly developed vaccines based on their professional medical knowledge. For non-HCWs, Popularization of medical general knowledge, such as of vaccine-preventable diseases, may improve the intention of non-HCWs to receive a COVID-19 vaccine.

\section{Data Sharing Statement}

The datasets used and analyzed during the current study are available from the corresponding author upon request.

\section{Ethics Approval and Consent to Participate}

The study was carried out in accordance with the guidelines laid down in the Declaration of Helsinki, and all procedures involving participants were conducted after an agreement was reached. The study protocol was approved by the Ethics Committee of the Wenzhou medical University, China (project approval number 2021-009). Participants were informed that 
their participation was voluntary, and consent was implied through their completion of the questionnaire.

\section{Acknowledgments}

The authors are grateful to the people who willingly participated in this study and to Wenzhou Medical University for its support.

\section{Funding}

This study was supported by grants from the Ministry of Education Humanities Social Sciences Research Project of China (No. 19JDSZ3049), Zhejiang Provincial Educational Science Planned Research Topics (No. 2019SCG162), the National Social Science Foundation Education Project (No. BIA180190), Wenzhou Science and Technology Bureau Basic Science Research Project (No. R2020020).

\section{Disclosure}

The authors declare that they have no competing interests.

\section{References}

1. World Health Organization. Coronavirus disease (COVID-19). Weekly epidemiological update; August 10, 2021 [cited August, 2021]. World Health Organization [Internet]. Available from: https://www.who.int/publications/m/item/weekly-epidemiologicalupdate-on-covid-19-10-august-2021. Accessed December 22, 2021.

2. Luke CJ, Subbarao K. Vaccines for pandemic influenza. Emerg Infect Dis. 2006;12(1):66-72. doi:10.3201/eid1201.051147

3. Reiter PL, Pennell ML, Katz ML. Acceptability of a COVID-19 vaccine among adults in the United States: how many people would get vaccinated? Vaccine. 2020;38(42):6500-6507. doi:10.1016/j. vaccine.2020.08.043

4. Bell S, Clarke R, Mounier-Jack S, Walker JL, Paterson P. Parents' and guardians' views on the acceptability of a future COVID-19 vaccine: a multi-methods study in England. Vaccine. 2020;38 (49):7789-7798. doi:10.1016/j.vaccine.2020.10.027

5. Lin Y, Hu Z, Zhao Q, Alias H, Danaee M, Wong LP. Understanding COVID-19 vaccine demand and hesitancy: a nationwide online survey in China. PLoS Negl Trop Dis. 2020;14(12):e0008961. doi:10.1371/journal.pntd.0008961

6. Mathieu E, Ritchie H, Ortiz-Ospina E, et al. A global database of COVID-19 vaccinations. Nat Hum Behav. 2021;5(7):947-953. doi:10.1038/s41562-021-01122-8

7. Larson HJ, Clarke RM, Jarrett $C$, et al. Measuring trust in vaccination: a systematic review. Hum Vaccin Immunother. 2018;14 (7):1599-1609. doi:10.1080/21645515.2018.1459252

8. Xiao X, Wong RM. Vaccine hesitancy and perceived behavioral control: a meta-analysis. Vaccine. 2020;38(33):5131-5138. doi:10.1016/j.vaccine.2020.04.076

9. Gidengil CA, Parker AM, Zikmund-Fisher BJ. Trends in risk perceptions and vaccination intentions: a longitudinal study of the first year of the H1N1 pandemic. Am J Public Health. 2012;102(4):672-679. doi:10.2105/AJPH.2011.300407

10. Setbon M, Raude J. Factors in vaccination intention against the pandemic influenza A/H1N1. Eur J Public Health. 2010;20 (5):490-494. doi:10.1093/eurpub/ckq054
11. Halpin C, Reid B. Attitudes and beliefs of healthcare workers about influenza vaccination. Nurs Older People. 2019;31(2):32-39. doi:10.7748/nop.2019.e1154

12. Wu S, Su J, Yang P, et al. Willingness to accept a future influenza A (H7N9) vaccine in Beijing, China. Vaccine. 2018;36(4):491-497. doi:10.1016/j.vaccine.2017.12.008

13. Alsuwaidi AR, Elbarazi I, Al-Hamad S, Aldhaheri R, Sheek-Hussein M, Narchi H. Vaccine hesitancy and its determinants among Arab parents: a cross-sectional survey in the United Arab Emirates. Hum Vaccin Immunother. 2020;16(12):3163-3169. doi:10.1080/ 21645515.2020.1753439

14. Lennon RP, Small ML, Smith RA, et al. Unique predictors of intended uptake of a COVID-19 vaccine in adults living in a Rural College Town in the United States. Am J Health Promot. 2021;8901171211026132. doi:10.1177/08901171211026132

15. Fu C, Wei Z, Pei S, Li S, Sun X, Liu P. Acceptance and preference for COVID-19 vaccination in health-care workers (HCWs). medRxiv. 2020. doi:10.1101/2020.04.09.20060103

16. Herzog R, Álvarez-pasquin MJ, Camino Díaz JLDB, Estrada JM, Gil Á. Are healthcare workers' intentions to vaccinate related to their knowledge, beliefs and attitudes? A systematic review. BMC Public Health. 2013;13(1):154. doi:10.1186/1471-2458-13-154

17. Alonso JAN, González PJB, Carbonell JCN. Analysis of factors influencing vaccine uptake: perspective from Spain. Vaccine. 2001;20(Suppl 1):S13-S15. doi:10.1016/S0264-410X(01)00300-0

18. Paterson P, Meurice F, Stanberry LR, Glismann S, Rosenthal SL, Larson HJ. Vaccine hesitancy and healthcare providers. Vaccine. 2016;34(52):6700-6706. doi:10.1016/j.vaccine.2016.10.042

19. Picchio CA, Carrasco MG, Sagué-Vilavella M, Rius C. Knowledge, attitudes and beliefs about vaccination in primary healthcare workers involved in the administration of systematic childhood vaccines, Barcelona, 2016/17. Euro Surveill. 2019;24(6):1800117. doi:10.2807/1560-7917.ES.2019.24.6.1800117

20. Barrière J, Vanjak D, Kriegel I, et al. Acceptance of the 2009 A(H1N1) influenza vaccine among hospital workers in two French cancer centers. Vaccine. 2010;28(43):7030-7074. doi:10.1016/j.vaccine.2010.08.021

21. Unroe KT, Evans R, Weaver L, Rusyniak D, Blackburn J. Willingness of long-term care staff to receive a COVID-19 vaccine: a single state survey. J Am Geriatr Soc. 2020;69(3):593-599.

22. Adelson JL, McCoach DB. Measuring the mathematical attitudes of elementary students: the effects of a 4-point or 5-point likert-type scale. Educ Psychol Meas. 2010;70(5):796-807. doi:10.1177/ 0013164410366694

23. Salali GD, Uysal MS. COVID-19 vaccine hesitancy is associated with beliefs on the origin of the novel coronavirus in the UK and Turkey. Psychol Med. 2020:1-3. doi:10.1017/S0033291720004067

24. Yoda T, Katsuyama H. Willingness to receive COVID-19 vaccination in Japan. Vaccines. 2021;9(1):48. doi:10.3390/vaccines9010048

25. Sathianathan S, Van Scoy LJ, Sakya SM, et al. Knowledge, perceptions, and preferred information sources related to COVID-19 among healthcare workers: results of a cross sectional survey. Am J Health Promot. 2021;35(5):633-636. doi:10.1177/0890117120982416

26. Assaf AM, Hammad EA, Haddadin RN. Influenza vaccination coverage rates, knowledge, attitudes, and beliefs in Jordan: a comprehensive study. Viral Immunol. 2016;29(9):516-525. doi:10.1089/vim.2015.0135

27. Barry M, Temsah MH, Alhuzaimi A, et al. COVID-19 vaccine confidence and hesitancy among health care workers: a cross-sectional survey from a MERS-CoV experienced nation. PLoS One. 2021;16(11):e0244415. doi:10.1371/journal.pone.0244415

28. Kahn C, Beasley D. More women than men in U.S. nervous about fast rollout of COVID vaccine, and that's a problem. Reuters/Ipsos poll | Reuters; 2020. Available from: https://www.usnews.com/news/ top-news/articles/2020-12-11/more-women-than-men-in-us-nervousabout-fast-rollout-of-covid-vaccine-and-thats-A-problem-reutersipsos-poll. Accessed December 22, 2021. 
29. Detoc M, Bruel S, Frappe P, Tardy B, Botelho-Nevers E, GagneuxBrunon A. Intention to participate in a COVID-19 vaccine clinical trial and to get vaccinated against COVID-19 in France during the pandemic. Vaccine. 2020;38(45):7002-7006. doi:10.1016/j. vaccine.2020.09.041

30. Deleanu D, Petricau C, Leru P, et al. Knowledge influences attitudes toward vaccination in Romania. Exp Ther Med. 2019;18 (6):5088-5094. doi:10.3892/etm.2019.8124

31. Ashok N, Krishnamurthy K, Singh K, Rahman S, Majumder MAA. High COVID-19 vaccine hesitancy among healthcare workers: should such a trend require closer attention by policymakers? Cureus. 2021;13(9):e17990. doi:10.7759/cureus.17990

32. Di Gennaro F, Murri R, Segala FV, et al. Attitudes towards anti-SARS-CoV2 vaccination among healthcare workers: results from a national survey in Italy. Viruses. 2021;13(3):371. doi: $10.3390 / \mathrm{v} 13030371$

33. Dror AA, Eisenbach N, Taiber S, et al. Vaccine hesitancy: the next challenge in the fight against COVID-19. Eur J Epidemiol. 2020;35 (8):775-779. doi:10.1007/s10654-020-00671-y
34. Kuter BJ, Offit PA, Poland GA. The development of COVID-19 vaccines in the United States: why and how so fast? Vaccine. 2021;39(18):2491-2495. doi:10.1016/j.vaccine.2021.03.077

35. Cerullo M. Many health care workers are refusing to get a COVID-19 vaccine. CBS News; 2021. Available from: https://www.cbsnews. com/news/covid-vaccine-health-care-worker-reluctance/. Accessed December 22, 2021.

36. Kreps S, Prasad S, Brownstein JS, et al. Factors associated with US adults' likelihood of accepting COVID-19 vaccination. JAMA Netw Open. 2020;3 (10):e2025594. doi:10.1001/jamanetworkopen.2020.25594

37. Faasse K, Newby J. Public Perceptions of COVID-19 in Australia: Perceived Risk, Knowledge, Health-Protective Behaviors, and Vaccine Intentions. Front Psychol. 2020;11. doi:10.3389/ fpsyg.2020.00011

38. Wang Z. Political trust in China: forms and causes. In: White L, editor. Legitimacy: Ambiguities of Political Success of Failure in East and Southeast Asia. Singapore: World Scientific; 2005:113-139.
Journal of Multidisciplinary Healthcare

\section{Publish your work in this journal}

The Journal of Multidisciplinary Healthcare is an international, peerreviewed open-access journal that aims to represent and publish research in healthcare areas delivered by practitioners of different disciplines. This includes studies and reviews conducted by multidisciplinary teams as well as research which evaluates the results or conduct of such teams or healthcare processes in general. The journal

\section{Dovepress}

covers a very wide range of areas and welcomes submissions from practitioners at all levels, from all over the world. The manuscript management system is completely online and includes a very quick and fair peer-review system. Visit http://www.dovepress.com/testimonials. php to read real quotes from published authors. 